BUTP-96/11

\title{
Efficient Simulation of Quantum Systems by Quantum Computers
}

\author{
Christof Zalka \\ Institut für theoretische Physik, Universität Bern, Switzerland
}

March 25, 1996

\begin{abstract}
We show that the time evolution of the wave function of a quantum mechanical many particle system can be implemented very efficiently on a quantum computer. The computational cost of such a simulation is comparable to the cost of a conventional simulation of the corresponding classical system. We then sketch how results of interest, like the energy spectrum of a system, can be obtained. We also indicate that ultimately the simulation of quantum field theory might be possible on large quantum computers.

We want to demonstrate that in principle various interesting things can be done. Actual applications will have to be worked out in detail also depending on what kind of quantum computer may be available one day...
\end{abstract}

\section{Quantum Computers (QCs)}

Quantum computers are still imaginary devices, but it is hoped that eventually the technical problems involved in their realization can be overcome [1, 2, 3, 田, 5, 6, 6. Quantum computers could solve some problems much faster than conventional computers. Most prominently, Peter Shor (1994) has given a "quantum algorithm" for factoring large integers in polynomial time [8] (see also [2]).

A $l$-bit quantum computer may be thought of consisting of $l$ two-state systems. Computations would be carried out by inducing unitary transformations of a few at a time of these quantum-bits (qubits). This may be done by exterior fields controlled from the outside. Upon observation at the end of the "unitary computation" the quantum computer would collapse into a state where each qubit is either a 0 or a 1 . The art of finding a "quantum algorithm" is to extract useful information from a few such runs.

The main technical problem in realizing QCs is, to prevent unwanted interactions with the environment during the calculation. Such interactions can cause decoherence, such that the $\mathrm{QC}$ is no longer in a pure quantum state. Here we consider an idealized QC which doesn't suffer from such problems. 


\section{Simulating Quantum Systems}

General ideas about using specially designed quantum systems to simulate other quantum systems have been published, e.g. by Feynman [9]. I present here an actual implementation of the simulation of quantum mechanical many particle systems on a general purpose QC 1. For every degree of freedom of the system we need a $l$-bit quantum register. This allows to store the whole (discretized) wave function as amplitudes of the "classical" states in the quantum computer. Already to store so much information would be impossible for a conventional computer. It is then shown that the time evolution for short time steps amounts essentially to a Fourier transformation which can be carried out very efficiently on a quantum computer 8 , 10, 11].

\subsection{Quantum Mechanical Particle in 1 Dimension}

First we discretize the wave function and impose periodic boundary conditions:

$$
a_{n}=\psi(n \Delta x) \quad a_{n+N}=a_{n} .
$$

After proper normalization we store these amplitudes in a $l$-bit quantum register:

$$
|\psi\rangle=\sum_{n=0}^{N-1} a_{n}|n\rangle \quad N=2^{l}
$$

Where $|n\rangle$ is the basis state corresponding to the binary representation of the number $n$. For short time steps $\Delta t$ the Greens function of the Schrödinger equation is approximately:

$$
G\left(x_{1}, x_{2}, \Delta t\right)=k e^{i \frac{m}{2} \frac{\left(x_{1}-x_{2}\right)^{2}}{\Delta t}-i V\left(x_{1}\right) \Delta t} .
$$

Applying this to the amplitudes $a_{n}$ is equivalent to transforming the basis states with the inverse transformation. Using proper normalization to make the transformation unitary, we get:

$$
|n\rangle \rightarrow \frac{1}{\sqrt{N}} \sum_{n^{\prime}=0}^{N-1} e^{-i \frac{m}{2} \frac{\left(n-n^{\prime}\right)^{2} \Delta x^{2}}{\Delta t}+i V(n \Delta x) \Delta t}\left|n^{\prime}\right\rangle .
$$

The crucial observation is that this corresponds to two diagonal unitary matrices plus a discrete Fourier transformation:

$$
|n\rangle \rightarrow \underbrace{\frac{1}{\sqrt{N}} e^{-i \frac{m}{2} \frac{n^{2} \Delta x^{2}}{\Delta t}+i V(n \Delta x) \Delta t}}_{\text {diagonal }} \underbrace{\sum_{n^{\prime}=0}^{N-1} e^{i m \frac{n n^{\prime} \Delta x^{2}}{\Delta t}}}_{\text {Fourier trafo }} \underbrace{\left(e^{-i \frac{m}{2} \frac{n^{\prime 2} \Delta x^{2}}{\Delta t}}\left|n^{\prime}\right\rangle\right)}_{\text {diagonal }}
$$

\footnotetext{
${ }^{1}$ Independently and at practically the same time as me, Steven Wiesner [12] has published a preprint concerning this, using a slightly different approach
} 
To be able to use the fast Fourier transformation algorithm (FFT), we chose the parameters such that:

$$
m \frac{\Delta x^{2}}{\Delta t}=A \frac{2 \pi}{N}, \quad A \quad \text { integer . }
$$

Provided the potential $V$ has been made periodic (with period $\mathrm{N}$ ), this also makes the whole phase factor periodic in $n$ and $n^{\prime}$, as is necessary for consistency. It is rather straightforward to implement the FFT algorithm on a quantum computer [8, 10, 11] such that it uses only about $l^{2} / 2$ computational steps (local unitary transformations). The diagonal unitary transformations of the type

$$
|n\rangle \rightarrow e^{i c F(n)}|n\rangle
$$

can be done with the following succession of steps:

$$
|n, 0\rangle \rightarrow|n, F(n)\rangle \quad ; \quad|F(n)\rangle \rightarrow e^{i c F(n)}|F(n)\rangle \quad ; \quad|n, F(n)\rangle \rightarrow|n, 0\rangle
$$

In the first and last step the vectors represent two quantum registers. The first step corresponds to parallel classical calculations. It can be carried out on a quantum computer with a somewhat higher cost than just one such calculation on a conventional computer (P. Shor [8]). As all transformations are unitary they can just as well be inverted, so that the last step is also possible. The second step is really a transformation of the form

$$
|n\rangle \rightarrow e^{i c n}|n\rangle
$$

which can be carried out in a straightforward manner in $l$ steps by transforming each bit separately by an appropriate transformation. Note that of course all these operations are carried out in parallel on all basis states which is why this is often called "quantum parallelism".

\subsection{Many Particles and Field Theory}

The generalization to many particles is straightforward. For $n$ particles in 3 dimensions we need $3 n$ quantum registers. Besides the above steps we need transformations corresponding to the coupling between particles and between the different degrees of freedom of the same particle. They are diagonal unitary transformations acting on several registers, e.g.:

$$
\left|n, n^{\prime}, n^{\prime \prime}\right\rangle \rightarrow e^{i c F\left(n, n^{\prime}, n^{\prime \prime}\right)}\left|n, n^{\prime}, n^{\prime \prime}\right\rangle \quad .
$$

Such a transformation can be carried out analogously to eq.(8). For identical particles the initial state of the quantum computer has to be chosen symmetric resp. antisymmetric. Also the generalization to more general Hamiltonian 
operators than in the previous paragraph is possible (e.g. with coupling to a magnetic field).

Also quantum field theory could be simulated, e.g. by discretizing the field as in lattice gauge theory (for a standard text see e.g. [15]). For a bosonic quantum field this would be equivalent to having 1 particle at each lattice point. At any rate, applications like lattice QCD would require large quantum computers with thousands of quantum bits, which are not going to be realizable soon.

\subsection{Fermionic Field Theories}

Fermionic quantum fields pose some problems, as their functional formulation (path integrals, wave functionals,...) involves anticommuting Grassmann numbers. There is no wave functional in terms of the usual complex numbers, so we have to look for a representation of the fermionic field operator-algebra on some other Hilbert space. One possibility is the Fock space, where we have for the $n$-particle sector a totally antisymmetric wave function of $n$ variables. Another possibility is to give the occupation number for each particle species at each lattice point. For fermions this can only be 0 or 1 and thus requires only 1 qubit.

I expect, that further difficulties which may arise with quantum fields can in principle be overcome, possibly along the same lines as it is done in lattice QCD.

\section{Other manipulations}

\subsection{Simulating a decay to obtain the ground state}

Often one is interested in the ground state of a quantum system, be this the ground state of a molecule, the vacuum of a field theory or a hadron stable under the strong interaction. I propose here to obtain the ground state essentially in the same way as it happens in reality, namely by letting some initial state decay. Decays happen because the instable particle is coupled to some other quantum system, like the electromagnetic field. It is important that this quantum system has a lot of different energy eigenvalues such that it can absorb the energy from

various transitions in the decaying system. For the simulation of a quantum computer we may take a collection of 2-level systems with energy gaps $\Delta E=$ $E_{0} 2^{-n}, n=0 \ldots l$ such that the whole system has practically a continuous energy spectrum. The coupling to the quantum system of interest may be done in various ways.

During the simulation of the decay, the auxiliary "energy-drain" system must periodically be reset to its ground state such that it is able to absorb more energy. As the study of simple systems shows, this does not necessarily in every step, but only in the average, lead to a reduction of the energy expectation 
value of the system of interest. For actual implementations one may have to think about an adequate "resetting" strategy, e.g. depending on the state the auxiliary system is found in upon observation just before each resetting. Also one may want to reduce the coupling towards the end of the decay simulation in order not to disturb the ground state of interest too much.

Actually it may be enough to simulate the decay until the few lowest lying states (which are of interest) have a large amplitude. Then one can follow the procedure of paragraph 4.2 to obtain one of the energy eigenstates.

\subsection{Putting a wave function on a Quantum Computer}

Here I sketch a method which in principle allows us to store a given wave function as an initial state on a QC. I demonstrate this for the case of a scalar quantum mechanical particle in 1 dimension. The generalization to multi dimensional wave functions is then rather straightforward. Here I show only how the absolute value of the wave function can be stored in a quantum register, as above I have already shown how the complex phase can later be introduced.

So we want to carry out the following transformation on a $l$ bit quantum register which initially is in the state $|0\rangle$ :

$$
|0\rangle \rightarrow k \sum_{n=0}^{2^{l}-1}\left|\psi\left(n \frac{L}{2^{l}}\right)\right||n\rangle \quad, \quad \text { where } \quad \psi(x+L)=\psi(x)
$$

and $k$ is an appropriate normalization constant. The idea now is to split the norm of the initial state (which is 1 , of course) $l$ times such that in the end we get the $2^{l}$ contributions for the single basis states $|n\rangle$. $^{2}$ (Imagine a binary tree with $l$ levels and $2^{l}$ leaves.) For this to work out correctly each split has to yield the right ratio. For this we must have an algorithm which can compute the integrals

$$
I_{i, k}=\int_{\frac{k}{2^{i}} L}^{\frac{k+1}{2^{i}} L}|\varphi(x)|^{2} d x \quad, \quad \text { for } \quad k=0 \ldots 2^{i}-1 \quad \text { and } \quad i=1 \ldots l
$$

Now the first split is realized by a real $\mathrm{O}(2)$ rotation acting on the most significant qubit of the quantum register, thus:

$$
|0, \ldots\rangle \rightarrow \sqrt{I_{1,0}}|0, \ldots\rangle+\sqrt{I_{1,1}}|1, \ldots\rangle
$$

Such an $\mathrm{O}(2)$ rotation of a qubit can be carried out analogously to the rephasing of an amplitude (see eq. (『) ) once the rotation angle $\varphi$ has been calculated:

\footnotetext{
${ }^{2}$ Note that the norm of mutually orthogonal substates is a useful notion in quantum computing because it is conserved under unitary transformations.
} 


$$
\sin \varphi=\sqrt{I_{1,1}}
$$

The subsequent calculations of angles and rotations are then carried out in quantum parallelism, thus:

$$
\begin{array}{r}
\left|b_{l}, b_{l-1}, \ldots b_{l-i}, 0, \ldots\right\rangle \rightarrow \cos (\varphi)\left|b_{l}, b_{l-1}, \ldots b_{l-i}, 0, \ldots\right\rangle+\sin (\varphi)\left|b_{l}, b_{l-1}, \ldots b_{l-i}, 1, \ldots\right\rangle \\
\text { with } \sin (\varphi)=\sqrt{I_{l-i+1, k}} \text { and } k=b_{l-i}+2 b_{l-i+1}+\ldots+2^{i} b^{l}
\end{array}
$$

So this happens simultaneously for all values of the $b$ 's (resp. of $k$ ).

The method given here to input a wave function to a $\mathrm{QC}$ is rather ambitious, as essentially it evaluates the function at every (discretized) point $x$. In practice a simpler sceme may do.

\section{Obtaining Results of Interest}

\subsection{Measuring positions of particles, resp. field strengths}

One can think of various ways how to obtain quantities of interest from a quantum computer simulation of a quantum system. Say we have some state on the $\mathrm{QC}$ which we want to analyze, like the vacuum or a 1-particle state of a field theory. One can now make a measurement of some field strength simply by observing the corresponding quantum register. By repeatedly observing some field values in different points one may in several runs of the QC get a statistical estimate of $n$-point functions. In particular, one may obtain the correlation length. To get $n$-point functions for space-time points with a timelike separation, one has to continue the simulation of the time evolution between observations of single quantum registers.

\subsection{Measuring arbitrary observables}

To study the measurement problem in quantum theory, J. von Neumann has proposed an idealized simple interaction [13, 14] between the quantum system and the part of the measuring apparatus that directly interacts with the system. For convenience this part of the apparatus can be chosen to be equivalent to a quantum mechanical particle in 1 dimension or, more precisely, its Hilbert space is spanned by the basis vectors

$$
|x\rangle \quad x \quad \text { real, and } \quad \hat{X}|x\rangle=x|x\rangle .
$$

The time evolution during the measurement of an observable $\hat{A}$ shall then be given by 


$$
\hat{U}(t)\left|\Psi_{a}\right\rangle|x\rangle=\left|\Psi_{a}\right\rangle|x+k \cdot a \cdot t\rangle \quad, \quad \text { with } \quad \hat{A}\left|\Psi_{a}\right\rangle=a\left|\Psi_{a}\right\rangle \quad,
$$

where $\left|\Psi_{a}\right\rangle$ is the state of the system. One easily verifies, that the Hamilton operator $\hat{H}=k \hat{P} \hat{A}$ leads to this time evolution. Here $\hat{P}$ is the usual momentum operator for the imaginary quantum mechanical particle, thus $[\hat{X}, \hat{P}]=i \hbar$.

Now we simply have to implement this time evolution on the QC. This can be expected to work out for any reasonable observable represented by the hermitian operator $\hat{A}$ which can be written in terms of the fundamental observables and their conjugate momenta.

Then, after simulating this "1. stage von Neumann measuring process", we can observe the auxiliary quantum register and should find a number proportional to an eigenvalue of $\hat{A}$. By repeating this, we get the spectrum of $\hat{A}$ and the weight of the various $\hat{A}$-eigenstates in the original system state. Also after each observation the system will be in an eigenstate of $\hat{A}$. Thus this is a way of obtaining e.g. the energy eigenstates.

\section{Conclusion}

I have demonstrated that on a large enough quantum computer various quantum theoretic quantities of interest could be calculated, which may be too hard to compute by a conventional computer. This is in particular true for strongly interacting field theories like QCD. However simulating quantum fields would require large quantum computers with many thousand qubits, which we don't know whether they can ever be built. Furthermore, here I have assumed to have an ideal QC which doesn't suffer from decoherence and can carry out unitary transformations with sufficient precision (as these are really analog operations).

\section{References}

[1] S. Lloyd, A Potentially Realizable Quantum Computer. Science 261, 1569 (1993)

[2] I. L. Chuang et al., Quantum Computers, Factoring and Decoherence. LAUR-95-241, quant-ph/9503007

[3] I. L. Chuang and Y. Yamamoto, A Simple Quantum Computer. quant$\mathrm{ph} / 9505011$ (1995)

[4] I. L. Chuang et al., Effects of Loss and Decoherence on a Simple Quantum Computer. quant-ph/9602018 (1996)

[5] A. Barenco, A Universal Two Bit Gate for Quantum Computation. quantph/9505016 (1995) 
[6] A. Barenco et. al., Elementary Gates for Quantum Computation. quant$\mathrm{ph} / 9503016(1995)$

[7] W.G. Unruh, Maintaining Coherence in Quantum Computers. hepth/9406058 (1994)

[8] P. Shor, Polynominal-Time Algorithms for Prime Factorization and Discrete Logarithms on a Quantum Computer. In Proc. 35th Annual Symposium on Foundations of Computer Science. IEEE Press, pp 124-134, Nov. 1994, quant-ph/9508027

[9] R. Feynman, Internat. J. Theoret. Phys., 21, pp 467-488 (1982)

[10] D. Coppersmith, IBM Research Report RC 19642 (1994)

[11] A. Ekert and R. Jozsa (1995), Rev. Mod. Phys., to appear

[12] S. Wiesner, Simulations of Many-Body Quantum Systems by a Quantum Computer. quant-ph/9603028 (1996)

[13] J. von Neumann, Mathematical Foundations of Quantum Mechanics, Princeton University Press, Princeton (1955)

[14] B. S. DeWitt, in Physics Today, September 1970

[15] I. Montvay, G. Münster, Quantum Fields on a Lattice, Cambridge University Press (1994) 